\title{
Is multisystem inflammatory syndrome in children on the Kawasaki syndrome spectrum?
}

\author{
Rae S.M. Yeung ${ }^{1}$ and Polly J. Ferguson ${ }^{2}$ \\ 'Department of Paediatrics, Immunology and Medical Science, University of Toronto and Hospital for Sick Children, Toronto, Ontario, Canada. ${ }^{2}$ Department of Pediatrics, University of lowa Carver College of \\ Medicine, lowa City, lowa, USA.
}

\begin{abstract}
An alarming increase in children presenting with fever, hyperinflammation, and multiorgan dysfunction frequently requiring intensive care has been observed after severe acute respiratory syndrome coronavirus 2 infection. The illness resembles Kawasaki disease (KD), with coronary dilatation and aneurysm occurring in some. However, the cardiovascular manifestations were typically on the severe end of the KD spectrum, with cardiogenic shock a common presentation together with other features. This led to defining a unique syndrome named multisystem inflammatory syndrome in children (MIS-C). In this issue of the $J C I$, Lee and Day-Lewis et al. and Diorio et al. explored the clinical profiles associated with coronavirus disease 2019 in children. We posit that while splitting MIS-C into a separate disease may aid clinical management decisions, lumping it into the KD pot may better serve to understand pathobiology.
\end{abstract}

syndrome in children (MIS-C). Common elements to all definitions are presence of prolonged fever, multiorgan dysfunction, and laboratory evidence of hyperinflammation. The CDC case definition includes age less than 21 years, fever, laboratory evidence of inflammation, severe illness requiring hospitalization, and multiorgan dysfunction (cardiac, renal, respiratory, hematologic, gastrointestinal, dermatologic, or neurological) without another cause, together with evidence of current or recent SARS-CoV-2 infection (8).

\section{Identifying features of MIS-C}

In this volume of the JCI, investigators from two large pediatric centers report the clinical and immunologic features of their patients with MIS-C that help us gain insight into the pathogenesis of this inflammatory phenotype. Lee and Day-Lewis et al. (9) performed a retrospective study of 28 patients with MIS-C and compared them with historic cohorts of KD and MAS. Diorio et al. (10) prospectively studied patients with MIS-C ( $n=$ 6), severe COVID-19 $(n=9)$, and milder (hospitalized) COVID-19 $(n=5)$. Both performed studies to try to identify features of MIS-C that would aid in its diagnosis $(9,10)$. As in previous reports, involvement of the cardiovascular, gastrointestinal, and mucocutaneous systems were common in MIS-C, with less-prominent respiratory symptoms than those found in COVID-19. Also, the cardiovascular features were found to be most dramatic in MIS- $\mathrm{C}$ and can lead to rapid decompensation. Myocardial dysfunction and/or shock was present in $53 \%$ and $83 \%$ of patients, respectively; ionotropes were needed in $25 \%$ and $83 \%$ of patients, respectively; and more than half the patients required intensive care. In both cohorts, cardiovascular features overlapped with those of $\mathrm{KD}$ with 25\% (7 of 28) in Boston and 66\% (4 of 6) in Philadelphia, fulfilling criteria for complete or incomplete KD. And while 
Intensity of immune response

\begin{tabular}{|c|c|c|c|c|}
\hline Gl symptoms & Arthritis & Aneurysm & MAS & Shock \\
\hline & & Fever & & \\
\hline
\end{tabular}

Figure 1. Heterogeneous spectrum of hyperinflammation in Kawasaki syndrome. Disease model proposes that the magnitude or kinetics of the immune response creates a continuum of the phenotypic severity in KD from mild organ involvement such as gastrointestinal symptoms (e.g., diarrhea, hydrops of the gallbladder) or arthritis, to severe life-threatening complications such as MAS and cardiogenic shock.

most groups reported that the majority of patients with MIS-C had been previously healthy, interestingly, two individuals with MIS-C from the Boston cohort had a past medical history of KD. Coronary artery involvement was detected in 7 of the 34 (20.6\%) patients with MIS-C reported in the two cohorts, which is lower than the reports in the KD literature and lower than the $35 \%$ in the historic KD cohort. Treatments for MIS-C have been derived from lessons learned in $\mathrm{KD}$ and are reflected in the management plan in both cohorts; most children received IVIG and/or corticosteroids, and some received anakinra, an IL-1 cytokine-blocking agent $(9,10)$. The clinical similarities and response to similar therapy opens the question of whether this syndrome is a unique disease or part of the KD spectrum.

\section{Environment-triggered hyperinflammation}

$\mathrm{KD}$ is thought to be an environment-triggered inflammatory response leading to systemic vasculitis. KD is truly a syndrome, with a wide range of clinical phenotypes. The common clinical findings in affected children include the diagnostic criteria of prolonged fever ( $>5$ days) plus at least four of the five principle disease features, which include nonexudative conjunctival injection, mucosal erythema, redness/edema of the hands and feet, cervical adenopathy, and rash (11). Cases of incomplete KD occur, in which prolonged fever occurs with fewer than four clinical criteria plus presence of supportive laboratory findings. KD predominantly affects the medium-sized arteries, with a predilection toward the coronary arteries. Cardiac manifestations in KD include coronary aneurysm/dilatation, aortic root dilatation, myocarditis, and cardiogenic shock (9). KD predominantly affects young children and has a monophasic course for most. The disease manifestation are the results of an overly robust immune response, and while most agree on an environmental trigger, the exact agent(s) remain debated. One working hypothesis is that $\mathrm{KD}$ is initiated by an infectious trigger. This hypothesis is supported by both a seasonality of disease that varies by country and areas within countries and the occurrence of epidemics of KD $(12,13)$. Viruses, including coronavirus infections, have been implicated in KD pathogenesis $(14,15)$. In accord with this data, SARS-CoV-2 can be added to the long list of infectious agents associated with KD. Genetics have also been implicated as there are reports of affected sibling pairs, affected parent-child duos, and racial differences, with the highest prevalence in Asian children. Several susceptibility genes have been implicated, including immune-related genes involved in $\mathrm{T}$ cell activation, B cell signaling, apoptosis, and transforming growth factor beta signaling (13). Other environmental factors have also been implicated, as there is an association with wind currents but not with particulate air pollution $(16,17)$.

Like KD, MIS-C is a syndrome complex with a wide spectrum of clinical phenotypes. A spectrum of COVID-19associated hyperinflammation syndromes have been proposed (6) with three clinical patterns along the hyperinflammation spectrum in MIS-C: shock, KD, and fever with inflammation. $\mathrm{KD}$, like MIS-C, is an infection-triggered hyperinflammatory syndrome with varying degrees of inflammation: cardiogenic shock and MAS at the severe end of the spectrum and a self-limited febrile illness at the mild end. So, although MIS-C resembles KD, the severity of cardiovascular involvement and other nonoverlapping features have led to splitting MIS-C into a separate disease rather than lumping it into the Kawasaki syndrome disease spectrum. The severity of cardiovascular involvement with myocardial dysfunction and shock are features that may distinguish MIS-C as a unique disease process versus KD. However, KD can present with shock. In 2009, Kanegaye et al. (18) noted an increasing number of patients at their center presenting with acute KD accompanied by shock and hypotension, and defined this severe presentation as Kawasaki disease shock syndrome (KDSS). In their cohort, nearly one-third had myocardial dysfunction (ejection fraction < 54\%), coronary artery abn ormalities (62\%), and were often resistant to IVIG (18). Subsequently, other groups reported similar presentations in their KD cohorts. A more robust immune response occurs in KDSS, which is characterized by higher blood inflammatory markers. One group reported significantly higher serum levels of IL-6, IL-10, and INF- $\gamma$ in their KDSS cohort $(n=27)$ compared with KD controls $(n=43)(19)$. The incidence of shock in KD ranges from $1.4 \%$ to $7 \%$ of all KD cases $(18,20)$. The largest study to date reported 138 KDSS cases in 9488 children with KD (1.5\%) in Taiwan (21). Our own experience in Toronto shows an incidence of $2 \%$ for KDSS. An important characteristic of KDSS is the multiorgan nature of the inflammatory disease (22). The majority of patients with KDSS present with abdominal symptoms, mirroring the predominant clinical features of MIS-C (23). Cardiovascular complications are dramatically increased in children with KDSS; 65\% were found to have coronary aneurysm (24).

Life-threatening complications of $\mathrm{KD}$, including KDSS and MAS, have been considered rare events in the past, but like shock, MAS is reported in $2 \%$ to $5 \%$ of children with KD (25). MAS has also been reported with MIS-C. MAS is the result of a cytokine storm that can occur in the setting of infection, rheumatologic disease, and malignancy. Hyperferritinemia, cytopenias, liver dysfunction, and coagulation abnormalities are common. Children with MAS are often treated with steroids, cytokine-blocking agents (including IL-1 and IL-6 blockers), IVIG, and in severe cases, chemotherapeutic agents such as etoposide. Interestingly, children presenting on the severe KD spectrum with MAS and/or KDSS are usually older and are boys, con- 
sistent with the demographics described in MIS-C. Immunologically, many roads can lead to MAS, with the common underlying theme of hyperinflammation. In the case of MIS-C, SARS-CoV-2 infection-triggered MAS is seen in both the acute infectious phase of disease and also in the postinfectious MIS-C phenotype.

Immunologic studies were done by both the Lee and Day-Lewis et al. and Diorio et al. groups to gain insight into the pathophysiology of the disease as well as to explore immunological biomarkers that could serve to differentiate MIS-C from KD, MAS, and COVID-19 infection. Elevation of the serum IL-6 and IL-10 was found in patients with MIS-C in both cohorts as well as in severe COVID-19, whereas IFN- $\gamma$ was elevated across all three cohorts (mild and severe hospitalized COVID-19 and MIS-C) in the Philadelphia group, but not as a prominent cytokine in the Boston MIS-C group. Diorio et al. reported that IL-10 plus TNF levels, RT-PCR viral cycle thresholds (Cts), and peripheral smear burr cells can help distinguish MIS-C from mild or severe COVID-19, whereas Lee and Day-Lewis et al. found that soluble IL-12, ferritin, IL-18, and CXCL9 elevations were higher in MAS compared with MIS-C $(9,10)$. Blood vessels are thought to be an important target of SARS-CoV-2-triggered inflammation, mirroring the immunopathology identified in KD. The endothelial inflammation and injury described in COVID-19 (26) and in $\mathrm{KD}$ is in accord with the finding of complement activation reported by the Philadelphia group.

\section{Lumping or splitting}

Characterizing the disease phenotype, both clinically and biologically, as described in the reports by Lee and Day-Lewis et al. and Diorio and colleagues, adds to the foundational work needed to better define this new syndrome. MIS-C came to attention with children presenting with dramatic and extreme clinical features, with the case definition reflecting early observations based on children with severe disease, a circular and self-fulfilling definition. With further investigation and time, we are likely to learn about the milder end of the disease spectrum. Defining clear boundaries and differences between the newly named MIS-C hyperinflammatory spectrum of phenotypes from pre-COVID KD and/or the acute infectious phase of SARS-CoV-2 infection may be important for clinical management purposes; guiding when to target the invading virus during acute infection and/or the body's subsequent immune response. From a pathobiology viewpoint, however, such distinction may be a disservice to fully appreciating the MIS-C disease spectrum, as some of the differences may be due to the magnitude or kinetics of the immune response (Figure 1). The same excellent arguments in support of lumping or splitting are echoed in MIS-C, as in other infection-triggered, immune-mediated diseases. The global pandemic has given us a unique opportunity to examine the immune response leading to the MIS-C/KD phenotype triggered by a single infectious agent simultaneously in multiple regions and genetic populations. Goodwill and generosity has characterized the international community's response to COVID-19, and MIS-C is no exception. This silver lining in the COVID cloud will hopefully launch rigorous and scientific approaches to data collection and analyses using harmonized definitions to establish international disease registries, biorepositories, and research programs to improve our understanding of this emerging syndrome.

\section{Acknowledgments}

RSMY is supported by the Hak-Ming and Deborah Chiu Chair in Paediatric Translational Research from the Hospital for Sick Children, University of Toronto. PJF is supported by the Marjorie K. Lamb Professorship from the University of Iowa Carver College of Medicine.

Address correspondence to: Polly J. Ferguson, 200 Hawkins Drive, 2001 Boyd Tower, Iowa City, Iowa 52242, USA. Phone: 319.467.5111; Email: polly-ferguson@ uiowa.edu.

1. Dong Y, et al. Epidemiology of COVID-19 among children in China. Pediatrics. 2020;145(6): e20200702.

2. Riphagen S, Gomez X, Gonzalez-Martinez C, Wilkinson N, Theocharis P. Hyperinflammatory shock in children during COVID-19 pandemic. Lancet. 2020;395(10237):1607-1608.

3. Verdoni L, et al. An outbreak of severe Kawasakilike disease at the Italian epicentre of the SARSCoV-2 epidemic: an observational cohort study. Lancet. 2020;395(10239):1771-1778.

4. Feldstein LR, et al. Multisystem inflammatory syndrome in U.S. children and adolescents.
N Engl J Med. 2020;383(4):334-346.

5. Dufort EM, et al. Multisystem inflammatory syndrome in children in New York State. $N$ Engl J Med. 2020;383(4):347-358.

6. Whittaker E, et al. Clinical characteristics of 58 children with a pediatric inflammatory multisystem syndrome temporally associated with SARSCoV-2 [published online June 8, 2020]. JAMA https://doi.org/10.1001/jama.2020.10369.

7. Royal College of Paediatrics and Child Health. Guidance paediatric multisystem inflammatory syndrome temporally associated with COVID-19 (PIMS).https://www.rcpch.ac.uk/resources/guidance-paediatric-multisystem-inflammatory-syndrome-temporally-associated-covid-19-pims. Accessed August 25, 2020.

8. CDC. Multisystem inflammatory syndrome in children (MIS-C) associated with coronavirus disease 2019 (COVID-19). https://emergency. cdc.gov/han/2020/han00432.asp. Updated May 14, 2020. Accessed August 25, 2020.

9. Lee PY, et al. Distinct clinical and immunological features of SARS-CoV-2-induced multisystem inflammatory syndrome in children. J Clin Invest. 2020;130(11):5942-5950.

10. Diorio C, et al. Multisystem inflammatory syndrome in children and COVID-19 are distinct presentations of SARS-CoV-2. J Clin Invest. 2020;130(11):5967-5975.

11. McCrindle BW, et al. Diagnosis, treatment, and long-term management of Kawasaki disease: a scientific statement for health professionals from the American Heart Association. Circulation. 2017;135(17):e927-e99.

12. Nakamura Y. Kawasaki disease: epidemiology and the lessons from it. Int J Rheum Dis. 2018;21(1):16-19.

13. Kumrah R, Vignesh P, Rawat A, Singh S. Immunogenetics of Kawasaki disease. Clin Rev Allergy Immunol. 2020;59(1):122-139.

14. Kitano N, Suzuki H, Takeuchi T. Patient age and the seasonal pattern of onset of Kawasaki's disease. N Engl J Med. 2018;378(21):2048-2049.

15. Shirato K, Imada Y, Kawase M, Nakagaki K, Matsuyama S, Taguchi F. Possible involvement of infection with human coronavirus 229E, but not NL63, in Kawasaki disease. JMed Virol. 2014;86(12):2146-2153.

16. Rodo X, et al. Tropospheric winds from northeastern China carry the etiologic agent of Kawasaki disease from its source to Japan. Proc Natl Acad Sci U S A. 2014;111(22):7952-7957.

17. Zeft AS, et al. Kawasaki disease and exposure to fine particulate air pollution. J Pediatr. 2016;177:179-183.

18. Kanegaye JT, et al. Recognition of a Kawasaki disease shock syndrome. Pediatrics. 2009;123(5):e783-9.

19. Li Y, et al. Kawasaki disease shock syndrome: clinical characteristics and possible use of IL-6, IL-10 and IFN- $\gamma$ as biomarkers for early recognition. Pediatr Rheumatol Online J. 2019;17(1):1.

20. Dominguez SR, Friedman K, Seewald R, Anderson MS, Willis L, Glode MP. Kawasaki disease in a pediatric intensive care unit: a case-control study. Pediatrics. 2008;122(4):e786-90.

21. Lin MT, Fu CM, Huang SK, Huang SC, Wu MH. Population-based study of Kawasaki disease 
shock syndrome in Taiwan. Pediatr Infect Dis J. 2013;32(12):1384-1386.

22. Gitiaux C, et al. Cerebral vasculitis in severe Kawasaki disease: early detection by magnetic resonance imaging and good outcome after intensive treatment. Dev Med Child Neurol. 2012;54(12):1160-1163.
23. Zulian F, et al. Acute surgical abdomen as presenting manifestation of Kawasaki disease. JPediatr. 2003;142(6):731-735.

24. Gamez-Gonzalez LB, et al. Kawasaki disease shock syndrome: Unique and severe subtype of Kawasaki disease. Pediatr Int. 2018;60(9):781-790.
25. Latino GA, Manlhiot C, Yeung RS, Chahal N, McCrindle BW. Macrophage activation syndrome in the acute phase of Kawasaki disease. J Pediatr Hematol Oncol. 2010;32(7):527-531.

26. Varga Z, et al. Endothelial cell infection and endotheliitis in COVID-19. Lancet. 2020;395(10234):1417-1418. 